\title{
Physical and geometrical hybrid design of two-layer and depth- chirped holographic image guide for see-through glass type head mounted display
}

\section{Toshiteru Nakamura, Yuzuru Takashima}

Toshiteru Nakamura, Yuzuru Takashima, "Physical and geometrical hybrid design of two-layer and depth-chirped holographic image guide for seethrough glass type head mounted display," Proc. SPIE 10757, Optical Data Storage 2018: Industrial Optical Devices and Systems, 107570D (14 September 2018); doi: 10.1117/12.2323239

SPIE Event: SPIE Optical Engineering + Applications, 2018, San Diego, California, United States 


\title{
Physical and geometrical hybrid design of two-layer and depth-chirped holographic image guide for see-through glass type head mounted display
}

\author{
Toshiteru Nakamura*a, Yuzuru Takashima ${ }^{\mathrm{b}}$ \\ ${ }^{a}$ Center for Technology Innovation - Electronics, Hitachi, Ltd., 292 Yabecho, Totsuka-ku,

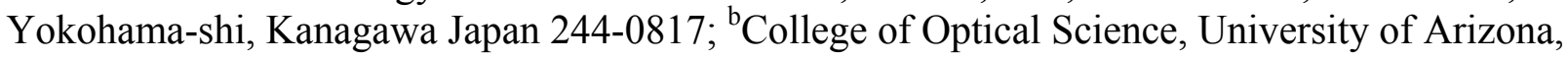 \\ 1630E. University Boulevard, Tucson, AZ USA 85721
}

\begin{abstract}
We designed the image guide with discretely depth-chirped holographic grating for head mounted display to equalize luminance over the Field of View (FOV) and increase throughput. To reduce the time required to optimize depth-chirped pattern, the mathematical optical efficiency prediction method was devised. The design approach enables, rapid turnaround in design process and precise prediction of optical performance of image guide incorporated depth-chirped grating. Display performance of the depth-chirped image guide identified by the mathematical optimization was verified by the geometrical and physical hybrid optical simulation that the RCWA code is integrated to geometrical ray trace code via DLL to incorporate effects of the diffraction. As a results, the design exhibited $315 \mathrm{~cd} / \mathrm{m}^{2} / \mathrm{lm}$ for the FOV $\left(35^{\circ}\right.$ $\left.(\mathrm{H}) \times 20^{\circ}(\mathrm{V})\right)$ and eye box size $( \pm 8.5 \mathrm{~mm}(\mathrm{H}), \pm 6.5 \mathrm{~mm}(\mathrm{~V}))$. The value of luminance was increased by $37 \%$ than unchirped image guide. Uniformity of luminance was further improved, from $33 \%$ to $47 \%$. In conclusion, we made clear the effect of depth-chirped image guide to increasing the performance of the image guide.
\end{abstract}

Keywords: Head mounted display, image guide, depth-chirped, uniformity of luminance

\section{INTRODUCTION}

There is a growing demand for Head Mount Displays (HMDs) in consumer and enterprise applications ${ }^{1-2}$. One of the common scenarios in the enterprise application, HMDs provides a work support by Augmented Reality (AR) technology. For the small form-factor optics and see-through function, image guides incorporating holograms as an input/output beam combiner and a pupil expander has been developed and widely investigated ${ }^{3-8}$. The thin flat plate with holographic element are placed in front of eye and creates the virtual image, thus suitable for a small eyeglass-shaped HMD module.

Although the functionality of the holographic image guide appears simple at a glance, in reality designing holographic image guide involves a rather complex optimization process to achieve uniform luminance of the virtual image over substantially wide Field of View (FOV) while minimizing loss of light during guiding light. In this paper, we propose incorporating a discretely depth-chirped surface relief hologram to optimize local diffraction efficiency according to FOV position to increase luminance throughput and its uniformity over the FOV. The hologram area is divided into sub areas where diffraction efficiency is individually controllable for example by varying grating depth. In the design process, first, we devised mathematical model of ray propagation focusing on energy splitting upon interaction of the ray with discretely depth-chirped hologram. By taking into account number of interactions with holograms and optical rays, the model is solved for throughput efficiencies of the depth-chirped image guide while using the values of pre-calculated diffraction efficiency by Rigorous Couple Wave Analysis (RCWA). The design approach enables, rapid turnaround in design process and precise prediction of optical performance by using depth-chirped pattern. The geometric (nonsequential ray trace) and ray-physical optics (RCWA) hybrid simulation are conducted to validate the depth-chirped pattern identified by the mathematical solution. The hybrid simulator verifies that for FOV of $40^{\circ}$, uniformity of $47 \%$ is feasible which improving throughput by factor of $37 \%$ compared to non-depth-chirped design.

Optical Data Storage 2018: Industrial Optical Devices and Systems, edited by Ryuichi Katayama,

Yuzuru Takashima, Proc. of SPIE Vol. 10757, 107570D · () 2018 SPIE

CCC code: $0277-786 X / 18 / \$ 18 \cdot$ doi: $10.1117 / 12.2323239$ 


\section{MULTI-LAYER IMAGE GUIDE DESIGN}

Figure 1 schematically depicts HMD optical system employing holographic elements at surface of the image guide. Generally three holographic elements are incorporated as surface relief gratings as Input, Vertical Expander (VE) and Output holograms ${ }^{4-7}$. Input hologram introduces signal light from an external optics such as projection lens with microdisplay into the image guide. The VE hologram expands vertical direction of eye-box. Finally, the Output hologram expands horizontal direction of eye-box and emit signal light to observer's pupil. In this configuration, grating vectors of each holograms $\left(K_{\text {Input }}, K_{V E}, K_{\text {Output }}\right)$ are arranged to satisfy the relationship: $K_{\text {Input }}+K_{V E}+K_{\text {Output }}=0$ so that light from output hologram has k-vector has the same magnitude of the k-vector of input signal light. Thus, HMD user can see a virtual image in their view. Once input rays interact with Input hologram, $1^{\text {st }}$ order diffraction on the Input hologram couples energy from air to guiding mode inside the glass slab. Inside the slab, the signal light propagates by total internal reflection (TIR). At the VE and Output hologram, similar process occurs. Light is redirected from VE (Output) hologram to Output hologram (Observer) by $1^{\text {st }}$ order diffraction while part of the energy is further delivered towards edge of the VE (Output) hologram by the TIR of $0^{\text {th }}$ order diffraction light.

(a)

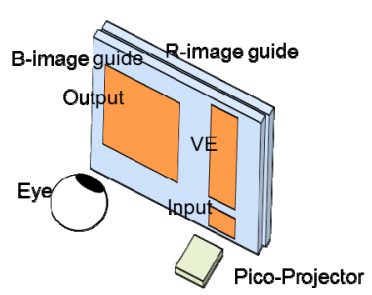

(b)

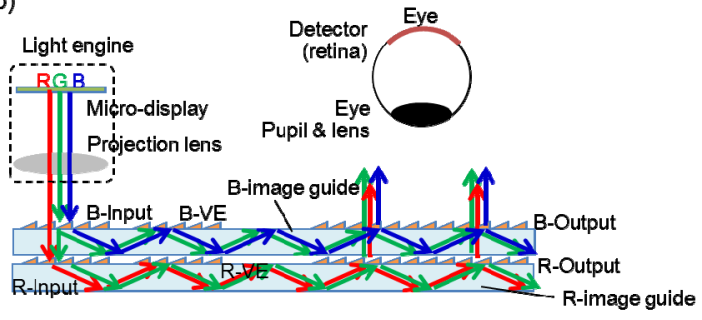

Fig. 1 (color online). (a) Schematic of image guide; (b) Throughput optical system of HMD using image guide, and solid color lines represent optical ray from micro-display which are colored corresponded to each signal color.

In general, multiple image guides are needed to propagate wide wavelength (RGB spectrum) because using a single layer image guide is a challenge due to variation of diffraction angle over wavelength range. In the design for angle of incidence (AOI) of light inside the image guide, the AOI of the light has to be larger than TIR angle criteria, so that TIR angle has to be as minimum limit of AOI of the light. On the other hand, in theory, the maximum allowable AOI is less than 90 degrees, though in reality, $<80$ degrees is a practical limit since light has to interact with VE and Output holograms multiple times. When blue and green light satisfy the required AOI range between TIR and $80^{\circ}$, red is difficult to satisfy the condition due to large AOI from large diffraction angle. To solve the problem, even higher $n_{d}>2.2$ is required. Unfortunately, the value $n_{d}>2.2$ exceeds $n_{d}$ of typical optical glass material. Based on the analysis, twolayer image guide are designed to separate propagation layer by wavelength, which has an image guide designated for blue (B-image guide), and designated for red (R-image guide). Green light which is wavelength between red and blue propagate both image guides. High index material (S-LAH88, Ohara glass, $n_{d}=1.91650$ ) is assumed as image guide material, and blazed or multi-level grating is feasible to fabricate by state of the art fabrication techniques ${ }^{9-10}$.

\section{DESIGN OF DEPTH-CHIRPED IMAGE GUIDE}

\subsection{Overview of depth-chirped image guide}

In case of the image guide as shown in Fig. 1, the image guide has common issue of uniformity of luminance at right and bottom side of FOV. This can be understood of the gradient of the luminance distribution schematically explained in Fig. 2. Signal ray forming left side of the image interacts hologram typically 10 or 20 times more than signal ray forming right side of the image, as a result, left side image loses luminance. In a similar way, upper side image loses luminance due to frequent interaction to the VE grating. 
(a)

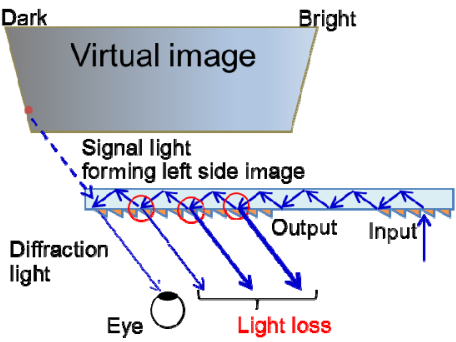

(b)

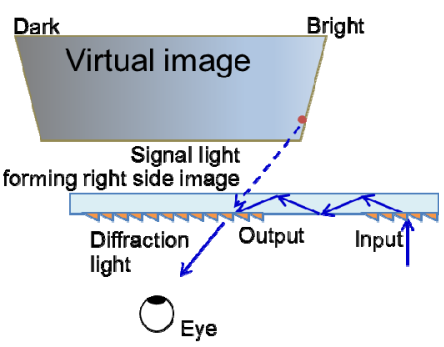

Fig. 2. Ray propagation process of signal light forming (a) left side image; (b) right side image.

To solve the problem of non-uniformity of the luminance over the FOV, we introduced depth-chirped grating to the image guide. In this design, the VE and Output grating are divided into multiple regions (sub-areas) having a different grating depth to control diffraction efficiency over the sub-areas (Fig. 3). At each of the sub areas grating depth is optimized to balance luminance over the FOV and increase overall optical throughput. Usually, HMD optical systems using the image guide involves both geometric and physical optics ray trajectory, and luminance of image is analyzed by geometrical optics while diffraction efficiency of holograms are incorporated there. The Rigorous Coupled-Wave Analysis (RCWA) method ${ }^{11-13}$ is used to calculate diffraction efficiency of hologram. However, in the geometric and physical optical analysis of the image guide, so many diffraction rays have to be traced, and it causes longer calculation time, for example about 10 hours a condition. That means difficulty to identify the best-solution of depth-chirped pattern. To overcome the computational time issue, we develop rapid optimization method is described in detail in this section.

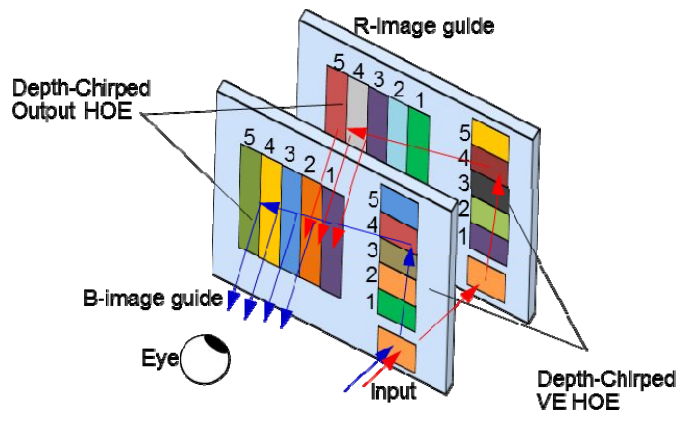

Fig. 3. Schematic of depth-chirped image guide.

\subsection{Mathematical model for the image guide with depth-chirped sub hologram areas}

We devised mathematical model of ray propagation focusing on energy splitting upon interaction of the ray with holograms for prediction of optical efficiency. A formulation of the developed optical efficiency prediction model considers critical factors for optical efficiency on the optical system from the light engine to user's pupil via image guide. Upon interaction of a ray to the Input hologram, a portion of power is taken away as a diffracted ray while rest of the power is preserved as a $0^{\text {th }}$ order diffraction. The process is repeated while ray interacts multiple times with sub areas of VE and Output hologram where diffraction efficiency at time of interaction is different among sub-areas.

(a)

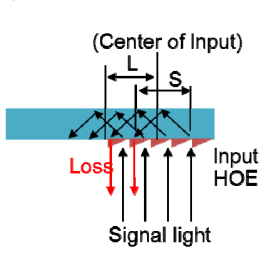

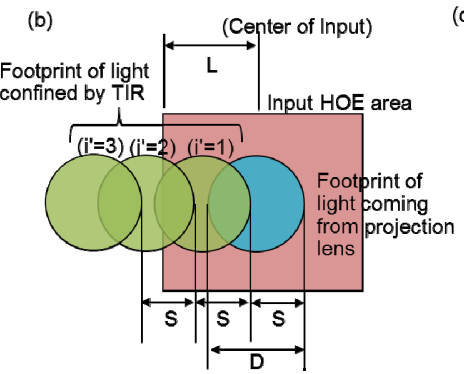

(c)

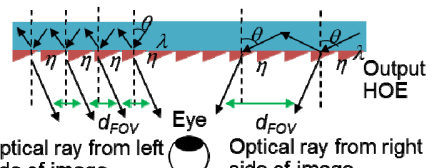

side of image

Fig. 4. Schematic of (a) light loss caused diffraction at back side Input hologram; (b) footprint of re-incident light at Input hologram; (c) fill factor of emitting ray from Output hologram. 
With the aid of Fig. 4, the process is mathematically formulated to calculate optical efficiency of ray through B-image guide along single ray $\left(f_{B}\right)$, and is given by,

$$
\begin{aligned}
f_{B} & =\eta_{B-\text { Input }}\left(d, \lambda, \theta, \phi, T, 1^{s t}\right) *(1-\beta) \\
& * \prod_{i=1}^{n} \eta_{B-V E}\left(d_{i}, \lambda, \theta, \phi, R, 0^{t h}\right)^{\left(m_{B-V E,}-1\right)} * \eta_{B-V E}\left(d_{k_{V E}}, \lambda, \theta, \phi, R, 1^{s t}\right) \\
& * \prod_{i=1}^{n} \eta_{B-\text { Output }}\left(d_{i}, \lambda, \theta, \phi, R, 0^{\text {th }}\right)^{\left(m_{B-O u t, i^{-1}}\right)} * \eta_{B-\text { Output }}\left(d_{k_{\text {Outout }}}, \lambda, \theta, \phi, T,,^{s t}\right) * \rho_{\lambda, B}
\end{aligned}
$$

In Eqn. (1), diffraction efficiency of each hologram is denoted as

$$
\eta_{\text {NameofGrating }}\left(d_{i}, \lambda, \theta, \phi, \text { Mode, DiffOrder }\right)
$$

the efficiency is a function of grating depth $d$, wavelength $\lambda$, AOI of $\theta$, and direction of grating $\phi$ of grating groove measured from horizontal direction of field of view. The subscripts "Mode" is either $T$ : Transmission, or R: Reflection, "DiffOrder" denotes order of diffraction, "Name of Grating" specifies kind of HOE such as B-VE is a VE hologram for Blue, and " $i$ " is an index of sub areas counted in ascending manner along ray propagation direction. The term in first line of Eqn. (1) denotes efficiency of ray when it interacts with B-Input hologram and coupled to B-image guide via $1^{\text {st }}$ order diffraction in transmission. As described in Fig. 5 (a), light loss due to re-incident to the back side of Input grating itself $(\beta)$ is given by Eqn. (3)

$$
\beta=\sum_{i^{\prime}=1}^{n}\left[\left(\frac{1}{2}-\frac{1}{\pi} \sin ^{-1} \frac{2\left(S_{i^{\prime}}-L\right)}{D}-\frac{2}{\pi D}\left(S_{i^{\prime}}-L\right) \cos \left(\sin ^{-1} \frac{2\left(S_{i^{\prime}}-L\right)}{D}\right)\right) *\left(\eta_{\text {Input }}\left(d, \lambda, \theta, \phi, R, 0^{t h}\right)^{i^{\prime}-1}-\eta_{\text {Input }}\left(d, \lambda, \theta, \phi, R, 0^{t h}\right)^{i^{\prime}}\right)\right]
$$

where ray traveling distance per a TIR is $S$, and half size of Input hologram is $L$ as shown in Fig. 5a and Fig. 5b.

The term in second line of Eqn. (1) calculates efficiency of single ray while it propagates on B-VE hologram area. Number of incidence to VE grating area for single ray is denoted as Eqn. (4)

$$
m_{\text {Name of grating, } i}
$$

The subscripts "Name of Grating" specifies kind of HOE such as B-VE is a VE hologram for Blue, and " $i$ " is an index of sub areas. The subscript " $k_{V E}$ " in Eqn. (1) indicates the sub area couples the ray to diffract toward to Output hologram, and its value varies with FOV.

The term in third line of Eqn. (1) denotes efficiency of ray when it interacts with B-Output hologram. In this line, the calculation incorporates reflection efficiency of $0^{\text {th }}$ order on B-Output, transmission diffraction efficiency of $1^{\text {st }}$ order on B-Output to emit the ray toward to the eye pupil, and the subscript " $k_{\text {Output }}$ " in Eqn. (1) indicates the sub area couples the ray to diffract toward to eye pupil, and its value varies with FOV. As mentioned above, the signal ray while reflecting in the image guide encounters VE and Output hologram again multiple times and it determine ray density at output hologram as a function of propagation angle (or correspondingly, function of FOV) is considered. (Fig. 5c). The fill factor of emitting ray $\left(\rho_{\lambda, \text { Layer }}\right.$. ) is calculated by Eqn. (5)

$$
\rho_{\lambda, \text { Layer }}=\sum_{-m}^{m} \sum_{-n}^{n}\left[2 D^{2} \cos ^{-1}\left(\frac{d_{m n}}{2 D}\right)-\frac{1}{2} d_{m n} \sqrt{4 D^{2}-d_{m n}^{2}}\right]
$$

where the exit pupil radius of projection lens is $R$, distance to adjacent emitting ray is $d$ (Fig. 5(b)). The subscript index of $\rho_{\lambda, \text { Layer }}$ specifies $\lambda$ is wavelength, and Layer such as B is an image guide layer for Blue.

Similarly, optical efficiency of ray through R-image guide along single ray $\left(f_{R}\right)$ is given by Eqn. (6)

$$
\begin{aligned}
& f_{R}=\eta_{B-\text { Input }}\left(d, \lambda, \theta, \phi, T, 0^{\text {th }}\right) * \eta_{R-\text { Input }}\left(d, \lambda, \theta, \phi, T, 1^{\text {st }}\right) *(1-\beta) \\
& * \prod_{i=1}^{n} \eta_{R-V E}\left(d_{i}, \lambda, \theta, \phi, R, 0^{t h}\right)^{\left(m_{\left.R-V E, i^{-1}\right)}\right.} * \eta_{R-V E}\left(d_{k_{V E}}, \lambda, \theta, \phi, R, 1^{s t}\right) \\
& * \prod_{i=1}^{n} \eta_{R-\text { Output }}\left(d_{i}, \lambda, \theta, \phi, R, 0^{\text {th }}\right)^{\left(m_{R} \text {-Output }, i^{-1)}\right.} * \eta_{R-\text { Output }}\left(d_{\text {kOutput }}, \lambda, \theta, \phi, T, 1^{\text {st }}\right) * \eta_{B-\text { Output }}\left(d_{\text {kOutput }}, \lambda, \theta, \phi, T, 0^{\text {th }}\right) * \rho_{\lambda, R}
\end{aligned}
$$

As signal ray goes to R-image guide transmit Input hologram of B-image guide, multi-layer structure is modeled in a following way. The transmitted $0^{\text {th }}$ order diffraction efficiency of B-image guide at Input hologram is considered (Fig. 
5a). Similarly, transmitted $0^{\text {th }}$ order diffraction efficiency of B-image guide at Output hologram is considered because signal ray come from R-image guide transmit Output hologram of B-image guide (Fig. 5b).

(a)

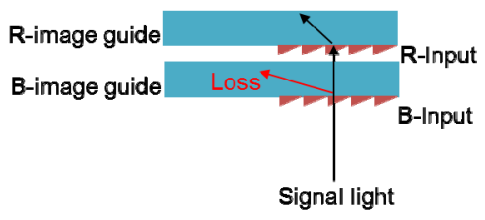

(b)

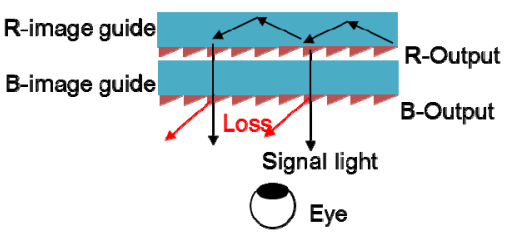

Fig. 5. Schematic of (a) light loss caused Input hologram of multi-layer structure; (b) light loss caused Input hologram of multi-layer structure.

Total optical efficiency of double layered image guide is given by Eqn. (7)

$$
f_{p}=f_{B}+f_{R}
$$

As a grating structure, a multi-level grating having 10 steps is assumed to approximate a blazed grating in this model. Diffraction efficiency is pre-calculated by RCWA as a function of AOI and wavelength, and polarization. In this model, diffraction efficiency is used as an average of p-polarized light and s-polarized light, so that the model has reprehensive grating depth diffraction efficiency $\eta$. If there is no matching condition data of grating depth, the model refers to the value of the adjacent data point and uses linear interpolation to estimate the diffraction efficiency of interest.

Using built rapid prediction method for optical efficiency, it is possible to conduct extensive search and optimize for depth-chirped grating within a practical time range. In this paper, the image guide model is assumed under condition of that FOV is 40 degrees $\left(35^{\circ}(\mathrm{H}) \times 20^{\circ}(\mathrm{V})\right)$ and eye-box size is $\mathrm{H}: \pm 8.5 \mathrm{~mm}, \mathrm{~V}: \pm 6.5 \mathrm{~mm}$. Size of VE and Output area are determined under consideration of eye-box size. Diameter of eye pupil is set $4 \mathrm{~mm}$, and thickness of image guide is set 1 $\mathrm{mm}$ a layer. Wavelength is considered $455 \mathrm{~nm}, 520 \mathrm{~nm}$, and $615 \mathrm{~nm}$. The optical efficiency is estimated in 9 points which are within the center of each block of $3 \times 3$ grid across the virtual image. The merit function for optimization evaluates uniformity of luminance along horizontal or vertical direction, and depth-chirped pattern is optimized to minimize a value of the merit function. VE/output area is divided to 5 sub-areas and optimized each area's grating depth. Since shallow or deep grating grooves are difficult to manufacture, grating depth of image guide are optimized from 40 $\mathrm{nm}$ to $200 \mathrm{~nm}$, and optical efficiency is constrained to exceed the efficiency of un-chirped image guide.

\section{PHYSICAL AND GEOMETRIC ANALYSIS}

\subsection{Physical and geometric hybrid simulation}

To evaluate accurately the image guide performance, the uniformity of luminance and power throughput of the discretely depth-chirped image guide which is identified by the developed optimization method is further simulated in the geometrical and physical hybrid optics simulator. The RCWA ${ }^{11-13}$ code is integrated to geometrical ray trace code (Zemax) to incorporate effects of the diffraction angle, grating depth, and polarization. In Fig. 6, a block diagram of the hybrid simulator is shown. In HMDs, light is guided though glass substrate by TIR and interacts with holograms multiple times. Upon each interaction with for example output coupling hologram, light is partially diffracted towards eye box. The hologram-light interaction condition are not unique, but varies over the field of view mainly due to variation of AOI and polarization state of ray with respect to grating vector of hologram, grating depth, and wavelength. To simulate such sequence of interactions a Dynamic Link Library (DLL) is developed to link RCWA code to geometrical ray trace code. As depicted in Fig. 6 DLL interfaces Zemax to lookup data created by RCWA code based on geometrical diffraction direction, polarization and electric field amplitude.

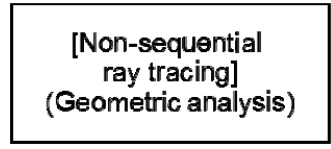

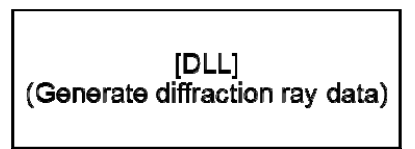

Hologram diffraction characteristic data Diffraction ray data

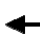

Fig. 6. Block diagram of physical and geometrical hybrid simulator 


\subsection{Analysis result of un-chirped image guide}

The performance of optimized image guides are verified by analysis by the above mentioned hybrid simulator. Light source is assumed as spectrum of the LEDs (Osram LCG H9RM, LEBA Q7WM) which has wide wavelength spectrum. The throughput optical system is shown as Fig. 1, and geometrical dimension of the image guide (thickness, hologram size, pupil size) is set same condition in the mentioned above.

First, the image guide having un-chirped (normal) hologram grating structure is analyzed. The results of luminance distribution of virtual image on each color shown in Fig. 7. In the graphs, the result of relative luminance at the each 9 evaluation points based on luminance of image center are shown. Virtual image of FOV is $35^{\circ}(\mathrm{H}) \times 20^{\circ}(\mathrm{V})$, and uniformity (Uniformity is determined by minimum relative luminance value at 9 evaluation points of screen based on evaluation point " 5 " in fig. $7 \mathrm{~g}$ ). and throughput is $33 \%$, and $230 \mathrm{~cd} / \mathrm{m}^{2}$, respectively. However, right side and bottom side of image tend to be high luminance and left side and upper side of image tend to be low luminance as mentioned in section 2 .

Luminance of virtual image by un-chirped image guide is calculated under condition of that each RGB LED light emitting duty is adjusted to display white color whose color coordinate is $\mathrm{x}=0.31, \mathrm{y}=0.34$ at center of virtual image. When luminous flux of signal light at the exit pupil of light engine is $1 \mathrm{~lm}$, luminance of image center of un-chirped image guide is $230 \mathrm{~cd} / \mathrm{m}^{2}$.

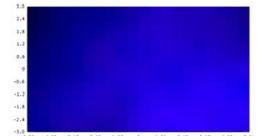

(a)

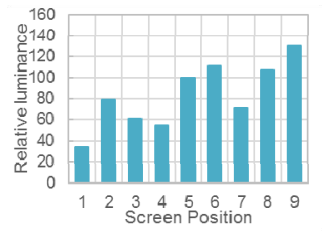

(d)

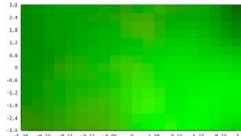

(b)

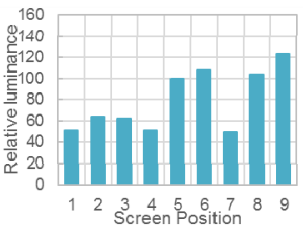

(e)

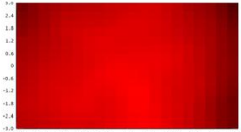

(c)

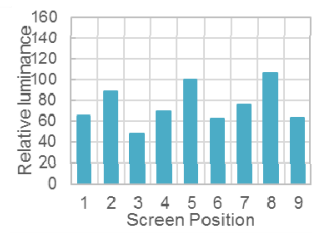

(f)

\begin{tabular}{|c|c|c|}
\hline (1) & (2) & (3) \\
\hline (4) & (5) & (6) \\
\hline (7) & (8) & (9) \\
\hline
\end{tabular}

(g)

Fig. 7. Simulation results of un-chirped image guide. Luminance distribution of virtual image on (a) blue LED; (b) green LED; (c) red LED; and (d) Relative luminance of each evaluation point based on image center on (d) blue LED; (e) green LED; and (f) red LED; (g) Evaluation point on the virtual image.

\subsection{Analysis result of depth-chirped image guide}

The simulation results of virtual image with image guide which is depth-chirped only in Output hologram (VE hologram is un-chirped.) is shown in Fig. 8. Luminance reaches to $280 \mathrm{~cd} / \mathrm{m}^{2} / \mathrm{lm}(\mathrm{x}=0.31, \mathrm{y}=0.34)$ with depth chirping grating at Output hologram when the light engine outputting $1 \mathrm{~lm}$ luminous flux as signal light at exit pupil, and luminance is increased by $22 \%$ than normal image guide structure. There is a change in luminance distribution using Output hologram's depth-chirped structure but uniformity of luminance is $35 \%$ that is almost same value as un-chirped image guide.

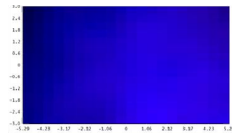

(a)

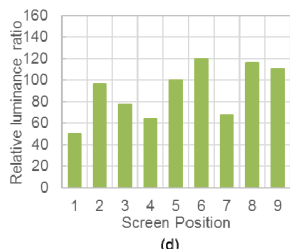

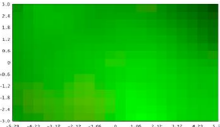

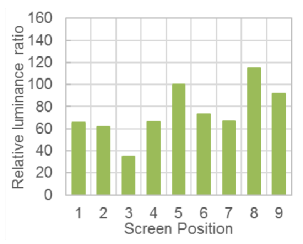

(e)

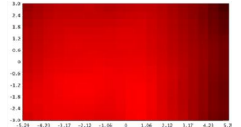

(c)

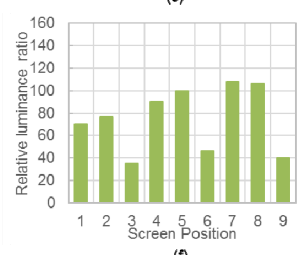

(f)

Fig. 8. Simulation results of depth-chirped image guide (VE is un-chirped). Luminance distribution of virtual image on (a) blue LED; (b) green LED; (c) red LED; Relative luminance of each evaluation point based on image center on (d) blue LED; (e) green LED; and (f) red LED. 
Compared to previous analysis results, the design result of the image guide which is depth-chirped Output and VE hologram is shown in Fig. 9. The results shows that the Output and VE hologram's depth-chirped structure improves uniformity of luminance from $33 \%$ to $47 \%$. Luminance at the image center reaches to $315 \mathrm{~cd} / \mathrm{m}^{2} / \mathrm{lm}(\mathrm{x}=0.31, \mathrm{y}=0.34)$ when the light engine outputting $1 \mathrm{~lm}$ luminous flux as signal light at exit pupil. Luminance is increased by $37 \%$ than the un-chirped image guide according to reducing the light loss in propagation process by the depth-chirped image guide.

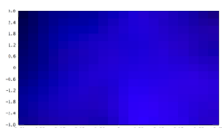

(a)

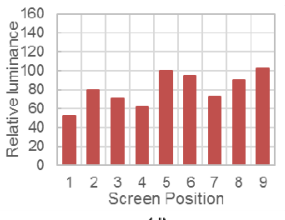

(d)

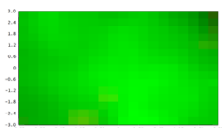

(b)

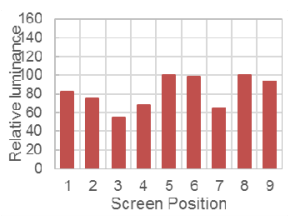

(e)

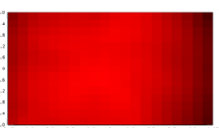

(c)

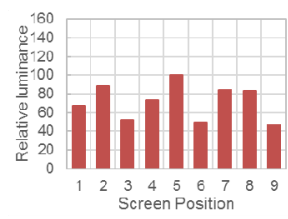

(f)

Fig. 9. Simulation results of depth-chirped image guide (VE, Output are depth-chirped). Luminance distribution of virtual image on (a) blue LED; (b) green LED; (c) red LED; Relative luminance of each evaluation point based on image center on (d) blue LED; (e) green LED; and (f) red LED.

Besides, considered actual grating characteristics calculated by RCWA has dependence of wavelength, AOI, groove direction and polarization. Also, actual grating has diffraction efficiency of higher diffraction order. These factors prevent to increase performance of image guide. To understand theoretical limit of the image guide through put, ideal grating which has only $0^{\text {th }}$ and $+1^{\text {st }}$ order diffraction and no dependence of wavelength, AOI and polarization are incorporated to the optimization model and its ideal depth-chirped image guide model is evaluated in geometric and physical analysis. In the result of ideal depth-chirped imageguide, luminance of virtual image reaches to $560 \mathrm{~cd} / \mathrm{m}^{2} / \mathrm{lm}$ $(\mathrm{x}=0.31, \mathrm{y}=0.34)$ when the light engine outputting $1 \mathrm{~lm}$ luminous flux as signal light at exit pupil.

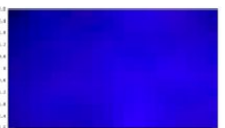

(a)

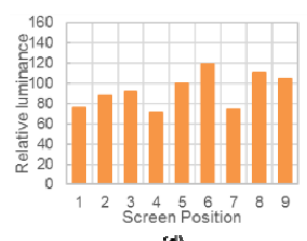

(d)

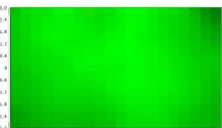

(b)

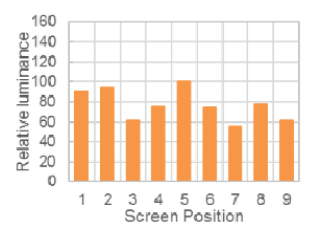

(e)

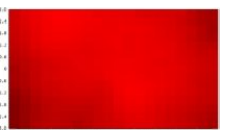

(c)

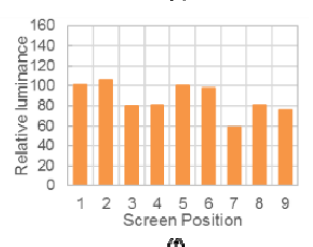

(1)

Fig. 10. Simulation results of depth-chirped image guide incorporated ideal grating. Luminance distribution of virtual image on (a) blue LED; (b) green LED; (c) red LED; Relative luminance of each evaluation point based on image center (position \#5) on (d) blue LED; (e) green LED; and (f) red LED.

Analysis results mentioned above by the hybrid simulator are tabulated in Table 1. In spite of the image guide which is depth-chirped only in Output hologram has an effect to increase optical throughput. Furthermore, the image guide which is depth-chirped in VE and Output hologram achieve higher performance for both of optical throughput and uniformity of luminance.

The proposed mathematical prediction method of optical efficiency for depth-chirped image guide reduces the time required to optimize grating depth of the image guide. The mathematical prediction method achieves to estimate optical efficiency for 1 or 2 seconds without tracing millions of optical rays instead of the geometrical and physical optical simulation it takes for about 10 hours to calculate virtual image. The depth-chirped image guide identified by the mathematical prediction method is verified by physical and geometric hybrid analysis that achieves to increase 
luminance and uniformity of luminance than un-chirped image guide. The proposed method is able to be applied to a specific design for depth-chirped image guides.

On the other hand, depth-chirped image guide using actual RCWA grating characteristic achieves $56 \%$ luminance of theoretical limit. Theoretical result imply luminance of image guide would increase about twice from new designed image guide. Due to actual grating has dependence of wavelength, AOI, groove direction and polarization and diffraction efficiency for higher diffraction order, these factors prevent to increase performance of image guide.

For the image guide mass production process, nanoimprint lithography technology may be easier way with low cost high through put. Its process requires lower refractive index of substrate for reasons to match refractive index of imprint resist for efficiency and spectral and AOI characteristics requirements. Incorporating three or more propagating layer to the image guide is going to be lower refractive index of the substrate, and multi-layer image guide will achieve higher uniformity of luminance.

Table 1. Analysis summary of optical performance of the image guides.

\begin{tabular}{|l|l|l|}
\hline \multicolumn{1}{|c|}{ Image guide } & \multicolumn{1}{|c|}{$\begin{array}{c}\text { Luminance } \\
\left(\mathbf{c d} / \mathbf{m}^{2} / \mathbf{l m}\right)\end{array}$} & \multicolumn{1}{|c|}{$\begin{array}{c}\text { Uniformity } \\
\mathbf{( \% )}\end{array}$} \\
\hline Un-chirped & 230 & 33 \\
\hline Depth-chirped (Only Output hologram) & 280 & 35 \\
\hline Depth-chirped (Output and VE hologram) & 315 & 47 \\
\hline Depth-chirped (Ideal hologram) & 560 & 55 \\
\hline
\end{tabular}

\section{CONCLUSIONS}

In this study, we designed depth-chirped image guide to equalize luminance and increase throughput over the FOV. The proposed mathematical optical efficiency prediction method for depth-chirped image guide reduces the time required to optimize grating depth of the image guide. The design approach enables, rapid turnaround in design process and precise prediction of optical performance by using depth-chirped pattern. Display performance of the depth-chirped image guide identified by the mathematical optimization was verified by the geometrical and physical hybrid optical simulation that the RCWA code is integrated to geometrical ray trace code via DLL to incorporate effects of the diffraction. The design exhibits $315 \mathrm{~cd} / \mathrm{m}^{2} / \mathrm{lm}$ for the FOV $\left(35^{\circ}(\mathrm{H}) \times 20^{\circ}(\mathrm{V})\right)$ and eye box size $( \pm 8.5 \mathrm{~mm}(\mathrm{H}), \pm 6.5 \mathrm{~mm}(\mathrm{~V}))$. This value of luminance at center of image is increased by $37 \%$ than conventional un-chirped image guide. Uniformity of luminance is further improved, from $33 \%$ to $47 \%$, by introducing depth-chirped grating. As a results of geometrical and physical optical analysis, we conclude that the depth-chirped image guide optimized by the developed prediction method effect to increase the performance of the image guide.

\section{REFERENCES}

[1] J. Lee, "Mobile AR in your pocket with google tango,"SID Int. Symp. Digest Tech. Papers 4(1), 17-18 (2017).

[2] D. Diakopoulos, and A. K. Bhowmik, "Project alloy: an all-in-one virtuan and merged reality platform," SID Int. Symp. Digest Tech. Papers 4(2), 19-22 (2017).

[3] M. Popovich, and S. Sagan, "Application specific integrated lensed for displays," SID Int. Symp. Digest Tech. Papers 45(1), 1060-1063 (2000).

[4] T. Levola, "Diffractive optics for virtual reality displays," J. Soc. Inf. Disp. 14, 467-475 (2006).

[5] T. Levola, and P. Laallonen, "Replicated slanted gratings with a high refractive index material for in and outcoupling of light," Opt. Express 15(5), 2067-2074 (2007).

[6] B. C. Kress, and W. J. Cummings, "Towards the ultimate mixed reality experience: HoloLens display architecture choices," SID Int. Symp. Digest Tech. Papers 11(1), 127-131 (2017). 
[7] D. Grey, and S. Talukdar, "Exit pupil expanding diffractive optical waveguide device," International Patent WO 2016/020643

[8] H. Mukawa, K. Akutsu, I. Matsumura, S. Nakano, T. Yoshida, M. Kuwahara, and K. Aiki, "A full-color eyewear display using planar waveguides with reflection volume holograms," J. Soc. Inf. Disp. 17/3, 185-193 (2009).

[9] T. Shiono, K. Setsune, O. Yamazaki, and K. Wasa, "Computer-controlled electron-beam writing system for thin film micro-optics," J. Vac. Sci. Technol. B., 5, 33-36 (1987).

[10] G. J. Swason, and W. B. Veldkamp, "Diffractive optical elements for use in infrared systems," Opt. Eng., 28, 605-608 (1989).

[11]H. Kogelnik, "Coupled wave theory for thick hologram gratings," The bell system Tech. J., 48(9), 2909-2947 (1969).

[12] M.G. Moharam, and T. K. Gaylord, "Three-dimensional vector coupled-wave analysis of planar-grating diffraction," J. Opt. Soc. Am., 73(9),1105-1112 (1983).

[13] M.G. Moharam, E. B. Grann, D. A. Pommet, and T. K. Gaylord, "Formulation for stable and efficient implementation of the rigorous coupled-wave analysis of binary gratings," J. Opt. Soc. Am., 12(5), 1068-1076 (1995). 